\title{
Implementación de una estrategia pedagógica para mejorar la atención en los estudiantes del grado cuarto de básica primaria en la institución educativa Carlos Pérez Escalante sede Marco Fidel Suárez ubicada en el municipio de Cúcuta Norte de Santander Colombia
}

Implementation of a pedagogical strategy to improve the attention of students of the fourth grade of primary school in the Carlos Pérez Escalante educational institution, Marco Fidel Suárez campus located in the municipality of Cúcuta Norte de Santander Colombia

Implementação de uma estratégia pedagógica para melhorar a atenção dos alunos do quarto ano do ensino fundamental na instituição educacional Carlos Pérez Escalante, campus Marco

Fidel Suárez, localizado no município de Cúcuta Norte de Santander, Colômbia

Resumen

En la presente investigación se implementó una estrategia pedagógica para mejorar la atención de los estudiantes del grado cuarto de la institución educativa Carlos Pérez Escalante sede Marco Fidel Suárez en el área de lengua castellana. Se utilizó para este proyecto la investigación con enfoque mixto, cualitativa y cuantitativa. De esta manera se obtuvo como resultados una muestra conformada por 38 estudiantes, de ambos sexos, con los cuales se logró diagnosticar las funciones cognitivas de la atención de los 
estudiantes, utilizando la plataforma CogniFit, en el aprendizaje cognitivo y se evidenció el fortalecimiento en el área de lengua castellana, de los estudiantes del grado cuarto de la institución educativa Carlos Pérez Escalante sede Marco Fidel Suárez. De acuerdo a las categorías de atención voluntaria y estrategias pedagógicas, se realizaron actividades de talleres teniendo los resultados de desempeño académico en el segundo periodo del año escolar, que hace parte de las pruebas internas de la institución, que a su vez los resultados fueron mejorados. La investigación permitió utilización de instrumentos, como estrategias pedagógica, que permitió estar a la vanguardia de nuevas tecnologías, con el programa de estimulación cognitiva de CogniFit de forma personalizada para fortalecer la atención y entretenimiento de los educandos. Para concluir se pudo evaluar en los estudiantes, la motivación, teniendo mayor habilidad en la enseñanza de aprendizaje, utilizando nuevos métodos didácticos, que deberían ser aplicados en todas las áreas.

Palabras claves: Aprendizaje, atención, cognitivo, CogniFit, estrategias, pedagógica.

\section{Abstract}

In the present research, a pedagogical strategy was implemented to improve the attention of the fourth grade students of the Carlos Pérez Escalante educational institution, Marco Fidel Suárez campus in the area of spanish language. The research with a mixed, qualitative and quantitative approach was used for this project. In this way, a sample was obtained consisting of 38 students, of both sexes, with whom it was possible to diagnose the cognitive functions of the attention of the students, using the CogniFit platform, in the cognitive learning and the strengthening was evidenced in the Spanish language area, of the fourth grade students of the Carlos Pérez Escalante Educational Institution, Marco Fidel Suárez campus. According to the categories of voluntary attention and pedagogical strategies, workshop activities were carried out having the results of academic performance in the second period of the school year, which is part of the internal tests of the Institution, which in turn improved the results The research allowed for the use of tools, such as pedagogical strategies, which made it possible to be at the forefront of new technologies, with the cognitive stimulation program of CogniFit in a personalized way to strengthen the care and entertainment of the students. To conclude it was possible to evaluate in the students, the motivation, having greater ability in teaching learning, using new didactic methods, which should be applied in all areas.

Keywords: Learning, attention, cognitive, CogniFit, strategies, pedagogical. 


\section{Resumo}

Nesta pesquisa estratégia pedagógica foi implementado para melhorar o atendimento para os alunos de quarta série da sede Escola Carlos Perez Escalante Marco Fidel Suárez na área de língua espanhola. A pesquisa com abordagem mista, qualitativa e quantitativa foi utilizada para este projeto. Assim foi obtido como resultado de uma amostra composta por 38 alunos, de ambos os sexos, com o qual foi possível diagnosticar as funções cognitivas da atenção dos alunos que utilizam a plataforma CogniFit, aprendizagem cognitiva e fortalecimento evidenciados na Área de língua espanhola, dos alunos do quarto ano do instituto educacional Carlos Pérez Escalante, campus Marco Fidel Suárez. De acordo com as categorias de estratégias de atenção e ensino voluntárias, atividades oficinas foram tomando os resultados de desempenho acadêmico na segunda metade do ano, que é parte do teste interno da instituição, que por sua vez resulta foram melhorados. A investigação usando ferramentas como estratégias pedagógicas que permitiu estar na vanguarda das novas tecnologias, com cognitiva programa de estimulação CogniFit para fortalecer atenção e de entretenimento alunos personalizadas. Para concluir foi possível avaliar nos alunos, a motivação, tendo maior habilidade no ensino de aprendizagem, utilizando novos métodos didáticos, que deveriam ser aplicados em todas as áreas.

Palavras-chave: Aprendizagem, atenção, cognitiva, cogniFit, estratégias pedagógicas.

\section{Justificación}

La presente investigación trabaja inicialmente el proceso de aprendizaje pedagógico, ya que, mediante la atención adecuada y oportuna, los facilitadores, podrán garantizar, una educación de calidad, la cual beneficia la adquisición de saberes para la vida y fortalece la capacidad de logros individuales; a través de la cultura, en la sociedad. De esta manera los docentes fortalecen los conocimientos en los estudiantes, a través de nuevas herramientas de enseñanza, y asimismo, desarrollar habilidades de manera ágil, en la institución educativa Carlos Pérez Escalante sede Marco Fidel Suárez, teniendo como objetivo mejorar el área de lengua castellana, en la cual han sido afectados los niveles de aprendizaje, por esto se crean estrategias pedagógicas que permitan al docente reflexionar los métodos utilizados e incorporar en su planificación de manera deliberada enseñanzas pedagógicas que permitan desarrollar las habilidades cognitivas en los estudiantes, para ello, es necesario un amplio proceso de formación por parte del docente en el cual pueda enriquecer cada día su práctica 
profesional, facilitando al alumno la posibilidad de potenciar sus habilidades de pensamiento y su formación integral.

Esta Investigación se apoyará en la plataforma CogniFit, la cual está formado por un completo equipo de profesionales especializados en el estudio de la plasticidad sináptica y procesos de neurogénesis. Este programa de estimulación cognitiva está personalizado para las necesidades de cada usuario. Se inicia por una precisa evaluación de la atención y otras funciones cognitivas fundamentales. Con base en los resultados de la evaluación, el programa de estimulación cognitiva de CogniFit ofrece de forma automatizada un entrenamiento personalizado para fortalecer la atención y otras funciones cognitivas que se consideren necesarias según la evaluación.

Esta Investigación se apoyará en la plataforma CogniFit, la cual está formado por un completo equipo de profesionales especializados en el estudio de la plasticidad sináptica y procesos de neurogénesis. Este programa de estimulación cognitiva está personalizado para las necesidades de cada usuario. Se inicia por una precisa evaluación de la atención y otras funciones cognitivas fundamentales. Con base en los resultados de la evaluación, el programa de estimulación cognitiva de CogniFit ofrece de forma automatizada un entrenamiento personalizado para fortalecer la atención y otras funciones cognitivas que se consideren necesarias según la evaluación.

\section{Atención CogniFit}

Plataforma CogniFit. ¿Qué es CogniFit? CogniFit es una empresa del sector salud, fundada en 1999 por el profesor Shlomo Breznitz, enfocada en el desarrollo y comercialización de productos online para la evaluación y mejora de la salud cognitiva. Actualmente CogniFit es un programa líder, reconocido y utilizado por la comunidad científica y compañías a nivel internacional.

¿Para qué puede servir? ¿Cuál es su objetivo último? Mediante los datos cognitivos obtenidos a través de la exploración y la normalización de resultados por edad y países, CogniFit entiende cuál es la situación cognitiva particular de cada individuo $y$ puede ofrecer un programa de ejercicio cerebral personalizado. Dicha metodología está detallada y patentada.

¿A través de esta herramienta se trabaja sobre dominios cognitivos concretos o sobre determinadas patologías? Las baterías de evaluación permiten evaluar más de 20 funciones cognitivas fundamentales, perfectamente definidas y sometidas a un control de medida objetivo que proporciona resultados.

¿Están los resultados de esta herramienta testados? Todas las 
herramientas de exploración y estimulación de las funciones cerebrales han sido probadas y validadas clínicamente. El producto está disponible en 18 idiomas, se trabaja con hospitales, universidades, colegios, fundaciones y centros de investigación de todo el mundo.

¿En qué consiste y como se desarrolla la evaluación de los estudiantes? ¿Qué tipo de datos se obtienen? Las baterías de evaluación constan de preguntas específicas necesarias para determinar aspectos particulares de los niños y niñas, así como de un número variable de tareas (test) en función de las habilidades cognitivas que se deseen evaluar.

Tras completar una evaluación, que típicamente dura entre 20 y 40 minutos el sistema genera un informe personalizado para cada usuario. Dicho informe incluye estimaciones de rangos de riesgos de sufrir ciertas patologías cognitivas, así como un detallado listado de puntuaciones de múltiples habilidades cognitivas comparadas con el grupo según la edad.

Los entrenamientos sin embargo son un complemento de estimulación cognitiva que no deben sustituir a otros elementos tan importantes como una buena hidratación, sueño, deporte e interacciones sociales de los estudiantes.

\section{Conclusiones}

Para concluir la investigación y con base a lo anterior expuesto, generando los resultados del desarrollo del objetivo general de implementar una estrategia pedagógica para mejorar la atención de los estudiantes del grado 403 de la Institución Educativa Carlos Pérez Escalante sede Marco Fidel Suárez en el área de Lengua Castellana, fueron satisfactorios como lo muestran los resultados de la evaluación interna del segundo periodo del año escolar.

Por otro lado, este proyecto se desarrolló con el grado cuarto y la muestra objeto de estudio, 403, esta población se caracteriza por ser flotante fronteriza vulnerable. La estrategia utilizada para diagnosticar los estudiantes fue la plataforma CogniFit, la cual permitió, conocer las debilidades en las funciones ejecutivas y luego fortalecerlas a través de talleres estructurados, teniendo en cuenta los referentes del Ministerio de Educación Nacional, proporcionando al educando un avance del conocimiento mejorando, y a su vez, la comunicación dentro del aula y de la misma.

El docente del área de Lengua castellana debe buscar motivar al niño con estrategias pedagógicas innovadoras, que los estudiantes se vean motivados a aprender jugando y con experiencias significativas cotidianas; también es importante en estas edades 
facilitar el aprendizaje cognitivo, el cual favorece el trabajo de manera eficaz, rápida y enriquecedora, educando a los estudiantes en valores de cooperación, responsabilidad, sensibilidad, pertinencia $y$ en el aprendizaje significativo donde combinan conocimientos, técnicas, estrategias, destrezas y experiencias.

Por otra parte, la implementación de una estrategia pedagógica por medio de la plataforma CogniFit, obtuvo buenos resultados, ya que permitió que los estudiantes, colocaran en práctica sus conocimientos, y de esta manera enriquecer el trabajo en equipo. Además se pudo evaluar en los estudiantes, la motivación.

\section{Referencias}

Alarcón Gallego, E., \& Guzmán Grijalva, M. (2016). Potenciar a atención y concentración de los estudiantesdel grado $2^{\circ}$ de la escuela Isabel de castilla a través de actividades artisticas y lúdico pedagógicas. Bogotá, Colombia: Fundación Universitaria Los Libertadores.

Allen, P. A., Sliwinski, M., Bowie, T., \& Madden, D. J. (2002). Differential age effects in semantic and episodic memory. Journal of Gerontology, 57, 173-186.

Alonso, J., Antó, J.M., \& Moreno, C. (1990). Spanish version of the NottinghamHealth Profile: translation and preliminary validity. Am J Public Health, 80,704-8.

Álvarez de Arcaya, A. Mª., \& Besga, B.

(2000).

Sombras.http//clic.xtec.net/pr ojects/sombras/jclic/sombras. jclic.zip.

Atkinson, R. C., \& Shiffrin, R. M. (1968). Human memory: A proposed system and its control processes. In K.W. Spence (Ed), The psychology of learning and motivation: advances in research and theory, (vol. 2, pp. 89-195). New York: Academic Press.

Baddeley, A. D. (1999). ¿Cuántas clases de memoria? Evidencias de la mcp. In A. Baddeley (Ed.), Memoria humana. Teoría y práctica. (pp. 33-56). Madrid: McGraw-Hill.

Baddeley, A. D. (1999). La atención y el control de la memoria. In A. Baddeley (Ed.), Memoria humana. Teoría y práctica (pp. 101-121). Madrid: McGrawHill.

Baddeley, A. D. (1999). La función de la memoria en la cognición: Memoria de trabajo. In A. Baddeley (Ed.), Memoria humana. Teoría y práctica (pp. 57- 81). Madrid: McGraw-Hill.

Baddeley, A. D. (1999). Percepción y recuerdo. In A. Baddeley (Ed.), Memoria humana. Teoría y práctica. (pp. 11-32). Madrid: McGraw-Hill. Baddeley, A. D., \& Hitch, G. (1974). Working memory. In G.A. Bower (Ed), 
Recent advances in learning and motivation, vol. 8. New York: Academic Press.

Bauermeister José J., (2012), Hiperactivo impulsivo distraído ¿Me conoces?. New York: Editorial The Guilford Press.

Bolívar Hernández, M. (2015). Diseño de una estrategia didáctica para el desarrollo de la funcion educativa a partir del estudio de os ecosistemas en el grado segundo de la básica primaria de la institución educativa Primitivo Leal La Doctora (Sabaneta, Ant). Medellín, Antioquia: Universidad Nacional de Colombia.

Bravo, N. H. (2008). Estrategias pedagógicas dinamizadoras del aprendizaje por competencias. Sinú, Colombia: Universidad del Sinú.

Castro Aguilera, M. F., Jiménez, A., González, D., \& Rincón, D. (2016. p.5-7). Atención. Institución Universitaria ECR, Bogota.

Cerda, H. (1992). El ejercicio investigativo a partir de la descripción de los aspectos más característicos, distintivos y particulares de las personas, situaciones o cosas, o sea, aquellas propiedades que las hacen reconocibles a los ojos de los demás.
Cognifit. (2018). CogniFit Inc. Obtenido de CogniFit (c) 2018: www.cognifit.com/es

De Walt K, \& De Walt B. (2002). idus.us.es. Obtenido de idus.us.es:

https://idus.us.es/xmlui/bitstr eam/handle/11441/40256/tes is\%20juan\%20carlos.pdf?seque nce $=1$

CogniFit. (2003). Mindfittm. Brain power fitness. Santander: 2E Sistema Recomind, S. L.

Colcombe, S., \& Kramer, A. F. (2003). Fitness effects on the cognitive function of older adults: A meta-analytic study. Psychological science, 14(2), 125-130. Cowan, N. (2001). The magical number 4 in shortterm memory: A reconsideration of mental storage capacity. Behavioral and Brain Sciences, 24(1), 87185.

Cowan, N., Wood, N. L., Wood, P. K., Keller, T. A., Nugent, L. D., \& Keller, C. V. (1998). Two separate verbal processing rates contributing to shortterm memory span. Journal of Experimental Psychology: General, 127, 141-160.

Elliott. (2000). Obtenido de http://www.redalyc.org/html/ 551/55121025012/

Elosua, M.R. (1992): "El aprendizaje significativo desde un enfoque social”, Revista de Psicología 
Universitas Tarraconensis, 2.716.

Giné, C. (coord) (2010). La educación inclusiva. De la exclusión a la plena participación de todo el alumnado. Barcelona: Editorial Horsori.

González, A. V. \& Ramírez, J. (2012). Estrategias pedagógicas alternativas en pausa para la inclusión educativa de escolares con discapacidad cognitiva. Tesis sin publicar. Universidad Tecnológica de Pereira, Colombia.

Hernández, O.(2011). Nivelemos 4. Lenguaje. Ministerio de Educación Nacional. Bogotá, Colombia.

Coll, C. (2007). Constructivismo en la escuela. Barcelona, España: Grao.

Julio Medrano, T., \& Pimentel Reyes, C. (2014). La atención en las niñas y niños en el nivel de preescolar de la institución educativa Ternera del distrito de Cartagena. Cartagena de Indias, Colombia: Universidad del Tolima Universidad de Cartagena.

Lerma Martorell, E. (2015). Propuesta de intervención con niños con trastorno de atención e hiperactividad para $1^{\circ}$ y $2^{\circ}$ de primaria. Valencia, España: Universidad Internacional de La Rioja.
Ley 115. (8 de Febrero de 1994). Obtenido de https://www.mineducacion.go v.co/1621/articles85906_archivo_pdf.pdf

Luria. (1975 p.2). La atencion: activando el aprendizaje. servicio publico de empleo.

Marvassio , M. D. (2014.p.13). Dificultades de atención en el aula aportes psicopedagogia. Tesis, Universidad Abierta Interamericana.

Mejía Rodríguez, G. (2017). Funciones Ejecutivas en niños y niñas de primaria: la imporntancia de las inteligencias múltiples como metodología de enseñanza aprendizaje . Bellaterra, Barcelona: Universidad Autónoma de Barcelona.

Men(2011). Nivelemos lenguaje $4^{\circ}$, guía del estudiante, Colombia.

Millán Jurado, M. (2012). Propuesta de metodología docente para alumnos con TDAH. Madrid, España: Universidad Internacional de La Rioja.

Obredor Ávila, C. (2015). Papel predictor de las funciones ejecutivas en el desempeño académico en las áreas de matemática y lenguaje en estudiantes de tercer grado de educación básica primaria. Barranquilla: Universidad de la Costa. 
Orjales (2014), Déficit de Atención sin Hiperactividad. Curso de inclusión educativa tercera edición presentada por el Centro de Altos Estudios Universitarios. Barcelona: Editorial CEPESL

Pino Melgarejo, M., \& Urrego Betancourt, Y. (2013). La importancia de las funciones ejecutivas para el desarrollo de las competencias ciudadanas en el contexto educativo. Cultura, educación y sociedad 4(1), 9-20, 9 - 20.

Pinto Númez, A. (2015). Estrategias didácticas para el fortalecimiento de la atención como pre requisito cognitivo en el desarrollo del aprendizaje autónomo con estudiantes de tercero de primaria de la institución educativa Técnico Agroindustrial El Espino. Duitama, Boyacá: Universidad Nacional Abierta y a Distancia UNAD.

Romero Moscoso, A., \& Callejas Cerquera, N. (2016). Programa "atento aprendo" como estrategia para mejorar la atención selectiva. Neiva, Huila: Corporación Universitaria Iberoamericana.

Salazar Celaya, D., \& Pérez Castañeda, M. (2014). Propuesta pedagógica para el mejoramiento de la práctica de los docentes de niños que presentan trastornos con déficit de atención con hiperactividad, en el primer grado de educación primaria, con apoyo en el modelo inclusionista. México, DF: Universidad Pedagógica Nacional.

Sánchez Valtierra, J. (2013). Práctica docente. Métodos de investigación mixto: un paradigma de investigación cuyo tiempo ha llegado.

Tashakkori, A. y Teddlie, C. [Eds.]. (2003). Handbook of mixed methods in social \& behavioral

Research [Manual de métodos mixtos en investigación social y del comportamiento].

Thousand Oaks: Sage Publications. Recuperado de http://books.google.co.cr/boo $\mathrm{ks}$ ?hl=es\&lr=\&id=F8BFOM8DC KoC\&oi=fnd\&pg $=$ PR9\&dq $=$ Han dbook+of+mixed+methods+in +social+\%26+behavioral+resea rch\&ots $=$ gSiQBBqzNk\&sig $=$ nFD Tw4B3zKb-_kP56vBzwZEEvC8 $\mathrm{v}=$ onepage $\& \mathrm{q} \& \mathrm{f}=$ false

Torre, J.C. (1992): Aprender a pensar y pensar para aprender. Estrategias de aprendizaje.

Madrid. MEC/Narcea.

Vásquez Vanegas, D. (2016). Propuesta metodológica para trabajar el déficit de atención con los niños de primero de básica de la unidad educativa Fray Vicente Soano sección vespertinaparalelo "A" en las edades de 5 y 6 años. Cuenca, Ecuador. 
Vidal-Abarca, E. Y Gilabert, R. (1991): Comprender para aprender. Un programa para mejorar la comprensión y el aprendizaje de textos. Madrid. Cepe.

Vosniadou, S. Y Ortony, A. (1989): similarity and analogical reasoning, New York.

Cambridge University Press.

Vygotsky, L.S. (1978): El desarrollo de los procesos psicológicos superiores. Barcelona, Crítica.

Weiert, F.E. Y Kluwe, R.H. (Eds.) (1987): Metacognition, motivation and understanding.

Hillsdale: N.J. Lea.

Wertsch, J. (Ed.) (1985): Culture, communication and cognition: vigotskian perspectives.

Cambridge, Ma: Cambridge University Press.

Yuste, C. Y Sánchez, J.M. (1990): Progresint. Programas Para La Estimulación De Las Habilidades De La Inteligencia. Madrid. Cepe.

Zimmerman, B.J. Y Schunk, D.H. (Eds.) (1989): Self-Regulated learning and academic achievement. Theory, research and practice. New York. Sringer-Verlag. 


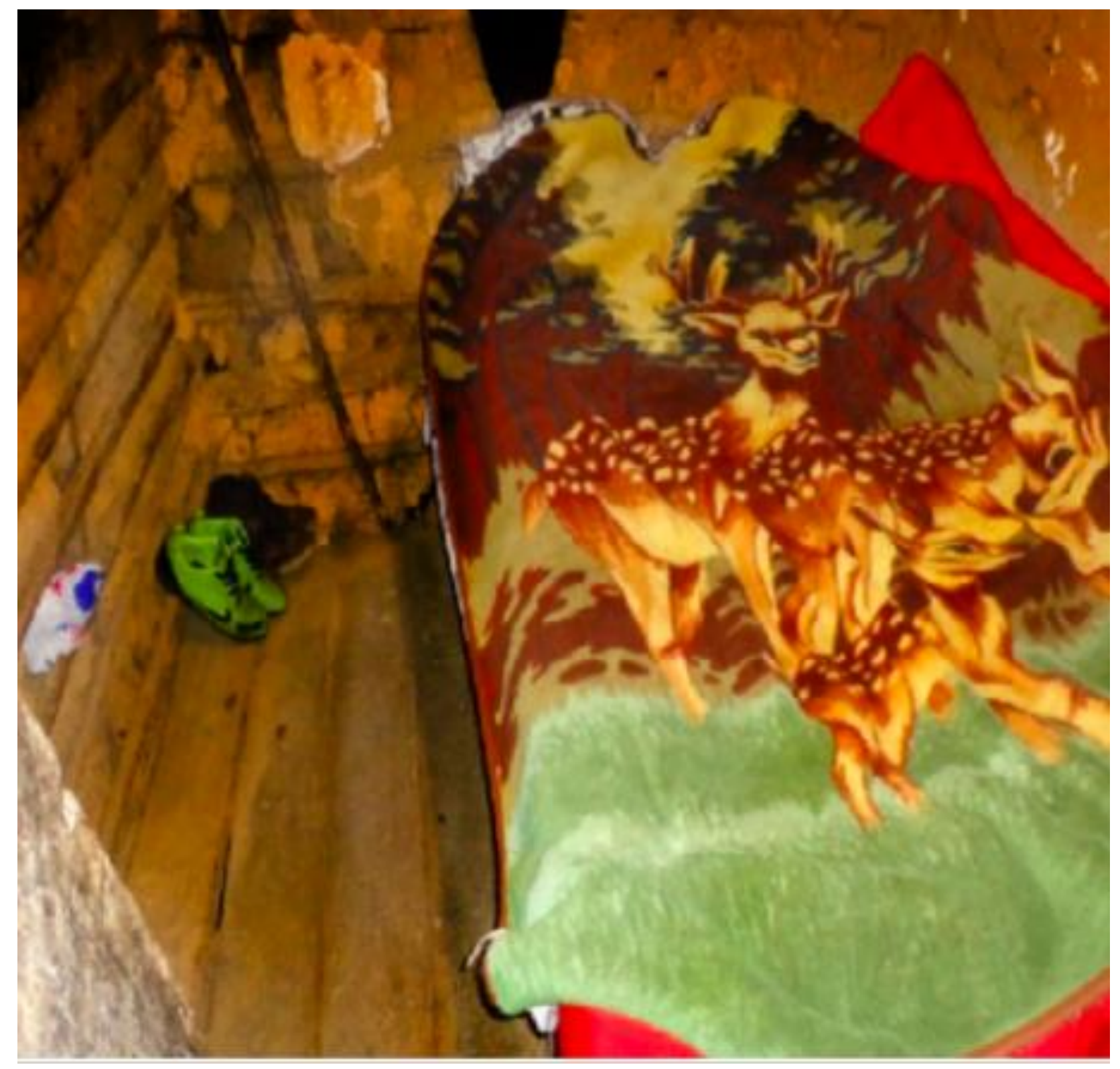

Número de la obra: 6

Título: "sobre camas"

Autor: Yenny Banesa Bonilla

Lugar: Zona rural municipio de Baraya

Finca: Mira Mundo

Fecha de captura: Febrero 28 de 2014

Técnica: Fotografía digital

Fuente: Bonilla, Y. B. (2014). Sobre camas. Una mirada estética popular del municipio de Baraya. Tesis de pregrado. Universidad Surcolombiana. Neiva, 2014. p.75. 